\title{
Complete Characterization of Multistep Methods with an Interval of Periodicity for Solving $y^{\prime \prime}=f(x, y)$
}

\section{By Rolf Jeltsch}

\begin{abstract}
Linear multistep methods for the second order differential equation $y^{\prime \prime}=$ $-\lambda^{2} y, \lambda$ real, are said to have an interval of periodicity if for a fixed $\lambda$ and a stepsize sufficiently small the numerical solution neither explodes nor decays. We give a very simple necessary and sufficient condition under which a linear multistep method has an interval of periodicity. This condition is then applied to multistep methods with an optimal error order.
\end{abstract}

1. Introduction. To solve numerically the initial value problem

$$
y^{\prime \prime}=f(x, y), \quad y(0)=\eta, \quad y^{\prime}(0)=\eta^{\prime},
$$

one uses linear multistep methods of the form

$$
\sum_{j=0}^{k} \alpha_{j} y_{n+j}=h^{2} \sum_{j=0}^{k} \beta_{j} f\left(x_{n+j}, y_{n+j}\right), \quad n=0,1,2, \ldots
$$

Here $h$ is the stepsize, and $x_{n}=n h, n=0,1,2, \ldots y_{n}$ will be an approximation to the exact solution $y(x)$ of (1) at $x=x_{n}$. The methods can be characterized by the polynomials

$$
\rho(\zeta):=\sum_{j=0}^{k} \alpha_{j} \zeta^{j}, \quad \sigma(\zeta):=\sum_{j=0}^{k} \beta_{j} \zeta^{j} .
$$

We shall always assume that the methods satisfy the following hypotheses:

(i) $\alpha_{j}, \beta_{j}$ are real for $j=0,1, \ldots, k$ and $\alpha_{k} \neq 0,\left|\alpha_{0}\right|+\left|\beta_{0}\right| \neq 0, \Sigma_{j=0}^{k}\left|\beta_{j}\right| \neq 0$.

(ii) $\rho(\zeta)$ and $\sigma(\zeta)$ have no common roots.

Henrici [2] has proved that the following two conditions are necessary and sufficient for convergence.

(iii) Condition of consistency:

$$
\rho(1)=\rho^{\prime}(1)=0, \quad \rho^{\prime \prime}(1)=2 \sigma(1) .
$$

(iv) Condition of stability: Let $\zeta_{j}$ be the roots of $\rho(\zeta)$. Then $\left|\zeta_{j}\right| \leqslant 1$; and if $\left|\zeta_{j}\right|=1$, then its multiplicity does not exceed 2 .

From (iii) and (iv) follows that 1 is a root of $\rho(\zeta)$ of exact multiplicity 2 . This root is called principal root and is denoted by $\zeta_{1}=\zeta_{2}=1$.

Consider the test equation

Received October 5, 1977.

AMS (MOS) subject classifications (1970). Primary $65 \mathrm{~L} 05$.

Key words and phrases. Linear multistep methods, second order differential equations, orbital stability, interval of periodicity, optimal methods, growth parameters. 


$$
y^{\prime \prime}=-\lambda^{2} y, \quad \lambda \in \mathbf{R}, \quad \lambda \neq 0,
$$

which has the nontrivial solutions

$$
y(x)=C_{1} e^{i \lambda x}+C_{2} e^{-i \lambda x}, \quad\left|C_{1}\right|+\left|C_{2}\right| \neq 0 .
$$

Applying (2) to (5) leads to

$$
\left[\rho(E)+H^{2} \sigma(E)\right] y_{n}=0, \quad n=0,1,2, \ldots .
$$

Here $E$ is the shift operator $E y_{n}=y_{n+1}$ and $H:=h \lambda$. (6) is a difference equation with constant coefficients. If

$$
\Phi(\zeta, H)=\rho(\zeta)+H^{2} \sigma(\zeta)
$$

is of degree $k$ in $\zeta$ and has only simple roots $\zeta_{j}(H)$ with $j=1,2, \ldots, k$, then the general solution of (6) has the form

$$
y_{n}=\sum_{j=1}^{k} D_{j} \zeta_{j}^{n}(H)
$$

Here $\zeta_{j}(H)$ are branches of the algebraic function $\zeta(H)$ given by

$$
\Phi(\zeta(H), H)=0 .
$$

Hence, $\zeta_{j}(0)$ are the roots of $\rho(\zeta)$. For brevity we shall use the notation $\zeta_{j}(0)=\zeta_{j}$. The exact solution of (5) neither decays nor explodes, as $x$ tends to infinity, but oscillates. One would like to have a similar behavior for the numerical solution as $n$ tends to infinity while $h$ is kept fixed. Lambert and Watson [4] have, therefore, introduced the notion of an interval of periodicity.

Definition. A method of type (2) satisfying (i)-(iv) is said to have an interval of periodicity $\left(0, H_{0}^{2}\right)$ if for all $H^{2} \in\left(0, H_{0}^{2}\right)$ one has

$$
\left|\zeta_{j}(H)\right|=1 \quad \text { for } j=1,2, \ldots, k \text {. }
$$

Lambert and Watson [4] have used a somewhat different definition. However, from their article it follows that the definitions are equivalent. It has been seen in [4] that a method with an interval of periodicity has to be symmetric, that is,

$$
\alpha_{k-j}=\alpha_{j}, \quad \beta_{k-j}=\beta_{j}, \quad j=0,1, \ldots, k .
$$

Moreover, if the principal root is the only double root of $\rho(\zeta)$ of modulus one then this necessary condition is also sufficient. In this note we first give a necessary and sufficient condition for a method to have an interval of periodicity which covers all cases. This result is then used to consider methods with optimal error order. A method is said to have error order $p$ if

$$
\rho(E) z(x)-\sigma(E) z^{\prime \prime}(x)=O\left(h^{p+2}\right)
$$

for all sufficiently smooth functions $z(x)$. In [2] it has been shown that for a convergent method of form (2) one has $p \leqslant k+2$. If $p=k+2$, then the method is called optimal. We shall show that these optimal methods have no interval of periodicity whenever $\zeta=-1$ is an essential root. It should be noted that the roots of $\rho(\zeta)$ and $\sigma(\zeta)$ have to occur in pairs of conjugate complex numbers. Moreover, a symmetric 
method cannot have an odd step number $k$ since (11) would imply $\rho(-1)=\sigma(-1)=$ 0 and thus (ii) is violated. For further properties of methods with an interval of periodicity and numerical applications we refer to the article by Lambert and Watson [4].

2. Statement of the Results. In the following we shall always assume that the method is symmetric. Hence from (11) follows that

$$
\rho(\zeta)=\zeta^{k} \rho(1 / \zeta)
$$

Thus, if $\zeta^{*}$ is a root of $p$, then $1 / \zeta^{*}$ is a root, too. Hence, the condition of stability (iv) implies that

$$
\left|\zeta_{j}\right|=1 \quad \text { for } j=1,2, \ldots, k \text {. }
$$

Assume that there are $2 d$ double roots of modulus 1 , each counted twice. We number these roots so that $\zeta_{1}=\zeta_{2}=1, \zeta_{3}=\zeta_{4}, \zeta_{5}=\zeta_{6}, \ldots, \zeta_{2 d-1}=\zeta_{2 d}$. These roots are called essential roots. The roots $\zeta_{j}$ with $2 d<j \leqslant k$ are all simple. Following Henrici [2], we associate with each essential root the growthparameter

$$
\mu_{j}:=\frac{2 \sigma\left(\zeta_{j}\right)}{\zeta_{j}^{2} \rho^{\prime \prime}\left(\zeta_{j}\right)}, \quad j=1,2, \ldots, 2 d .
$$

THEOREM 1. Assume that the method given by (2) satisfies (i)-(iv) and is symmetric. Then the method has an interval of periodicity if and only if all growthparameters are positive.

Remarks. 1. The condition of consistency implies that $\mu_{1}=1$.

2. From (13) and (14) follows that the growthparameter belonging to conjugate complex roots are equal and, thus, $\mu_{j}=\bar{\mu}_{j}$. Hence, a method has an interval of periodicity if and only if

$$
\mu_{2 j+1}>0 \text { for those } j=1,2, \ldots, d-1 \text { with } \operatorname{Im} \zeta_{2 j+1} \geqslant 0 \text {. }
$$

3. It is possible to give a similar theory for linear multistep method of the form

$$
\sum_{j=0}^{k} \alpha_{j} y_{n+j}=h \sum_{j=0}^{k} \beta_{j} f\left(x_{n+j}, y_{n+j}\right)
$$

to solve the initial value problem $y^{\prime}=f(x, y), y(0)=\eta$. However, for these methods to have an interval of periodicity the growthparameter does not come into play. In fact, a convergent method of form (17) which satisfies (i), (ii) has an interval of periodicity if and only if

$$
\alpha_{k-j}=\alpha_{j}, \quad \beta_{k-j}=-\beta_{j}, \quad j=0,1, \ldots, k,
$$

see Jeltsch [3].

In order to apply Theorem 1 to optimal methods we shall need the following theorem due to Henrici [2, p. 326].

Theorem 2. Assume that the method given by (2) satisfies (i)-(iv), is optimal and $\zeta_{2 d-1}=\zeta_{2 d}=-1$ is an essential root. Then 


$$
\mu_{2 d} \leqslant-1 / 15 \text {. }
$$

On combining Theorems 1 and 2, we find

THEOREM 3. Assume that the method given by (2) satisfies (i)-(iv), is optimal and has the essential root $\zeta=-1$. Then the method has no interval of periodicity.

Sometimes it is more convenient to use the variable $z$ defined by

$$
z=\frac{\zeta-1}{\zeta+1}, \quad \zeta=\frac{1+z}{1-z} .
$$

This transformation maps the unit disk of the $\zeta$-plane onto the left-hand plane $\operatorname{Re} z<0$. Let

$$
r(z):=(1-z)^{k} \rho\left(\frac{1+z}{1-z}\right)
$$

and

$$
s(z):=(1-z)^{k} \sigma\left(\frac{1+z}{1-z}\right) .
$$

Conversely, one has

$$
\rho(\zeta)=\left(\frac{\zeta+1}{2}\right)^{k} r(z)
$$

A similar formula holds for $\sigma(\zeta)$. From (19) it follows immediately that $r(z)$ is an even polynomial. The same is true for $s(z)$. Let us consider

$$
\begin{aligned}
& R(\tau)=a_{0}+a_{1} \tau+\ldots+a_{k / 2} \tau^{k / 2}:=r\left(\tau^{1 / 2}\right), \\
& S(\tau)=b_{0}+b_{1} \tau+\ldots+b_{k / 2} \tau^{k / 2}:=s\left(\tau^{1 / 2}\right) .
\end{aligned}
$$

Theorem 1 can be expressed in terms of $R(\tau)$ and $S(\tau)$.

Corollary. Assume that the method given by (2) satisfies (i)-(iv) and is symmetric. Let $\tau_{j}, j=1,2, \ldots, \delta$, be the double roots of $R(\tau)$. Then the method has an interval of periodicity if and only if

$$
R^{\prime \prime}\left(\tau_{j}\right) / S\left(\tau_{j}\right)<0 \quad \text { for } j=1,2, \ldots, \delta,
$$

and

$$
a_{k / 2-1} / b_{k / 2}>0 \quad \text { if } a_{k / 2}=0
$$

Remark 4. The corollary may be sometimes more convenient because $R(\tau)$ is a polynomial of degree $k / 2$, at most, while $\rho(\tau)$ has degree $k$. Moreover, all roots of $R(\tau)$ are real and nonpositive. Thus, the condition (24) can be checked using real numbers only.

Before we proceed with the proofs we shall give some examples. Lambert and Watson [4] introduced the following examples

$$
\begin{array}{ll}
\rho(\zeta)=(\zeta-1)^{2}\left(\zeta^{2}-\zeta+1\right)^{2}, & \sigma(\zeta)=1 / 2\left(\zeta^{4}+\zeta^{2}\right), \\
\hat{\rho}(\zeta)=\rho(\zeta), & \hat{\sigma}(\zeta)=1 / 2\left(\zeta^{5}+\zeta\right) .
\end{array}
$$


Both methods have error order 2, are symmetric and satisfy (i)-(iv). $\rho(\zeta)$ has double roots at $1, e^{i \pi / 3}$ and $e^{-i \pi / 3}$. Because of Remark 1 and Remark 2 we have to calculate the growthparameter $\mu_{3}$ belonging to $e^{i \pi / 3}$ only. One easily finds $\rho^{\prime \prime}\left(e^{i \pi / 3}\right)=$ $-6 e^{i 4 \pi / 3}, \sigma\left(e^{i \pi / 3}\right)=-1 / 2$ and $\mu_{3}=1 / 6>0$. Hence, (16) is satisfied and thus the method has an interval of periodicity. In the example (26), however, one finds $\hat{\sigma}\left(e^{i \pi / 3}\right)=1 / 2$ and $\mu_{3}=-1 / 6<0$. Thus, the method given by (26) has no interval of periodicity. In order to apply the corollary one determines the polynomials $R, \hat{R}$ and $S$. By a simple calculation one finds

$$
\begin{array}{ll}
R(\tau)=4 \tau(3 \tau+1)^{2}, & S(\tau)=\tau^{3}-\tau^{2}-\tau+1, \\
\hat{R}(\tau)=R(\tau), & \hat{S}(\tau)=-\tau^{3}-5 \tau^{2}+5 \tau+1 .
\end{array}
$$

In both examples one has $\delta=1$ and $\tau_{1}=-1 / 3$. From $R^{\prime \prime}\left(\tau_{1}\right)=\hat{R}^{\prime \prime}\left(\tau_{1}\right)=-24$, $S\left(\tau_{1}\right)=32 / 27$ and $\hat{S}\left(\tau_{1}\right)=-2 / 27$ one finds by the corollary exactly the same results for the methods (25), (26) as we have found before. As an illustration to Theorem 3, consider the following family of optimal four step methods given in [4]

$$
\begin{gathered}
\rho(\zeta)=(\zeta-1)^{2}\left(\zeta^{2}-2 \zeta \cos \phi+1\right), \\
\beta_{4}=\beta_{0}=(9+\cos \phi) / 120, \quad \beta_{3}=\beta_{1}=(13-3 \cos \phi) / 15 \\
\beta_{2}=(7-97 \cos \phi) / 60
\end{gathered}
$$

$\rho(\zeta)$ has the roots 1,1 and $e^{ \pm i \phi}$. Hence, the method (29) satisfies (i)-(iv) for $\phi \in$ $(0, \pi]$. According to Theorem 1 , the method has an interval of periodicity for $\phi \in(0, \pi)$, since $e^{ \pm i \phi}$ are simple roots, but has no interval of periodicity for $\phi=$ $\pi$, since -1 is an essential root. This result is confirmed by the results in [4] . Lambert and Watson have computed the length of the interval of periodicity and found

$$
H_{0}^{2}=60 \frac{1+\cos \phi}{11+9 \cos \phi} .
$$

One sees very nicely how $H_{0}$ shrinks as $\phi$ approaches $\pi$.

\section{Proof of the Results.}

Proof of Theorem 1. We have to show that $\left|\zeta_{j}(H)\right|=1$ for $H^{2} \in\left(0, H_{0}^{2}\right)$ if and only if (16) holds. Consider first a nonessential branch $\zeta_{j}(H)$, that is $\zeta_{j}(0)$ is a simple root of $\rho(\zeta)$. Observe that (11) implies

$$
\Phi(\zeta, H)=\zeta^{k} \Phi\left(\zeta^{-1}, H\right) .
$$

Hence, if $\zeta_{j}(H)$ is a branch of $\zeta(H)$, then $1 / \bar{\zeta}_{j}(H)$ is a branch too. Assume this branch is $\zeta_{i}(H)$; then one has for some $\hat{H}, \hat{H}>0$,

$$
\zeta_{i}(H)=1 / \xi_{j}(H) \quad \text { for } H \in[0, \hat{H}] .
$$

From $\left|\zeta_{j}(0)\right|=1$ and (30), it follows that

$$
\zeta_{j}(0)=1 / \zeta_{j}(0)=\zeta_{i}(0) \text {. }
$$


Assume that $i \neq j$. Hence, two different branches, $\zeta_{i}(H)$ and $\zeta_{j}(H)$, have the same value at $H=0$. Hence, $\zeta_{j}(0)$ is a double root of $\rho$. This is a contradiction to our initial assumption that $\zeta_{j}(0)$ be a nonessential root. Thus

$$
\zeta_{j}(H)=1 / \bar{\zeta}_{j}(H) \quad \text { for } H \in[0, \hat{H})
$$

for some $\hat{H}>0$, sufficiently small. We have, therefore, that the condition (10) is satisfied by all nonessential roots independently whether (16) holds or not. We consider now the essential branches $\zeta_{j}(H), j=1,2,3, \ldots, 2 d . \zeta_{j}(0)$ is a double root of $\rho(\zeta)$; and hence, the branch $\zeta_{j}(H)$ can be expanded into a convergent series in powers of $\eta^{1 / 2}$, where $\eta:=H^{2}$, see Ahlfors [1, p. 226]. Hence, $\zeta_{j}(H)$ is analytic in a neighborhood of $H=0$. Here we allow $H$ to be a complex variable. Through implicit differentiation one finds

$$
\left.\frac{d \zeta_{j}(H)}{d H}\right|_{H=0}=\sqrt{\frac{-2 \sigma\left(\zeta_{j}\right)}{\rho^{\prime \prime}\left(\zeta_{j}\right)}} \neq 0 .
$$

Hence, each map $H \rightarrow \zeta_{j}(H)$ can be inverted in a neighborhood of $\zeta_{j}$. One finds from (7) and (9) explicitly

$$
H^{2}=f(\zeta):=-\frac{\rho(\zeta)}{\sigma(\zeta)}
$$

Thus, the branch $\zeta_{j}(H)$ satisfies $\left|\zeta_{j}(H)\right|=1$ for $H \in\left[0, H^{\prime}\right]$ if and only if the function

$$
g_{j}(\phi):=f\left(\zeta_{j} e^{i \phi}\right)
$$

maps a sufficiently small interval $(-r, r), r>0$, onto an interval $\left[0, H^{\prime \prime}\right] \cdot g_{j}(\phi)$ is a real valued function of a real variable $\phi$ with a root at $\phi=0$ of exact multiplicity 2. Hence, $g_{j}(\phi)$ is positive in a neighborhood of $\phi=0$ if and only if $g_{j}^{\prime \prime}(0)>0$. However, an easy calculation reveals that

$$
g_{j}^{\prime \prime}(0)=\frac{\zeta_{j}^{2} \rho^{\prime \prime}\left(\zeta_{j}\right)}{\sigma\left(\zeta_{j}\right)}=\frac{2}{\mu_{j}}, \quad j=1,2, \ldots, 2 d .
$$

Proof of Corollary 1. Combining (22) and (23) leads to

$$
\rho(\zeta)=\left(\frac{\zeta+1}{2}\right)^{k} R(\tau),
$$

where

$$
\tau=\left(\frac{\zeta-1}{\zeta+1}\right)^{2}
$$

Similarly, one has

$$
\sigma(\zeta)=\left(\frac{\zeta+1}{2}\right)^{k} S(\tau)
$$


We obtain from letting $\zeta \rightarrow-1$ in (32)

$$
a_{k / 2}=\rho(-1) .
$$

Hence, if $\zeta=-1$ is not an essential root, then $R(\tau)$ has exact degree $k / 2$. To treat the case where $\zeta=-1$ is an essential root we differentiate (32) and obtain

$$
\begin{aligned}
2^{k} \rho^{\prime \prime}(\zeta)= & k(k-1)(\zeta+1)^{k-2} R(\tau) \\
& +8(k \zeta-\zeta-k+2)(\zeta+1)^{k-4} R^{\prime}(\tau) \\
& +16(\zeta-1)^{2}(\zeta+1)^{k-6} R^{\prime \prime}(\tau)
\end{aligned}
$$

Again, let $\zeta \rightarrow-1$ in (36). This gives

$$
a_{k / 2-1}=2 \rho^{\prime \prime}(-1) \neq 0 .
$$

Hence, if $\zeta=-1$ is an essential root, then $R(\tau)$ has exact degree $k / 2-1$. The transformation (33) maps the unit circle $|\zeta|=1$ onto the negative real line. In particular, $\zeta=1$ is mapped onto $\tau=0$. Hence, $\rho(\zeta)$ has a double root at $\zeta=1$ if and only if $R(\tau)$ has a simple root at $\tau=0$. A pair of roots $\zeta_{j}, \bar{\zeta}_{j}$ of $\rho(\zeta)$ which are different from \pm 1 are mapped onto the same negative real number $\tau^{\prime} . \tau^{\prime}$ is a simple (double) root of $R(\tau)$ if and only if $\zeta_{j}, \bar{\zeta}_{j}$ have been simple (double) roots. Hence, there is a one-to-one correspondence between pairs of essential roots $\zeta_{j}, \bar{\zeta}_{j}$ different from \pm 1 and the double roots $\tau_{i}, i=1,2, \ldots, \delta$, of $R(\tau)$. Let $\zeta_{j} \neq \pm 1$ and $\tau_{i}$ be its corresponding root of $R(\tau)$. Then $R\left(\tau_{i}\right)=R^{\prime}\left(\tau_{i}\right)=0$ and we find using (15), (34) and (36)

$$
\mu_{j}=\frac{\left(\zeta_{j}+1\right)^{4} S\left(\tau_{i}\right)}{8 \tau_{i} \zeta_{j}^{2} R^{\prime \prime}\left(\tau_{i}\right)}=\frac{1}{8 \tau_{i}} \frac{S\left(\tau_{i}\right)}{R^{\prime \prime}\left(\tau_{i}\right)}
$$

In the last equality we used $\left|\zeta_{j}\right|=1$. Since $\tau_{i}<0$, we see that $\mu_{j}>0$ is equivalent to (24a). If $\zeta_{j}=-1$, we obtain from letting $\zeta \rightarrow-1$ in (34) that $\sigma(-1)=b_{k / 2}$. This together with (15) and (37) gives

$$
\mu_{j}=4 b_{k / 2} / a_{k / 2-1}
$$

Hence, $\mu_{j}>0$ is equivalent to $(24 b)$.

Institute of Mathematics

Ruhr-University Bochum

D-4630 Bochum, Federal Republic of Germany

1. L. K. AHLFORS, Complex Analysis, McGraw-Hill, New York, 1953.

2. P. HENRICI, Discrete Variable Methods for Ordinary Differential Equations, Wiley, New York, 1962.

3. R. JELTSCH, Multistep Multiderivative Methods for the Numerical Solution of Initial Value Problems of Ordinary Differential Equations, Seminar Notes 1975-76, Dept. of Mathematics, University of Kentucky, 1976.

4. J. D. LAMBERT \& I. A. WATSON, "Symmetric multistep methods for periodic initial value problems," J. Inst. Math. Appl., v. 18, 1976, pp. 189-202. 